\title{
Selective drug trace detection with low-field NMR
}

\author{
Stefan Glöggler, ${ }^{* a}$ Meike Emondts, ${ }^{a}$ Johannes Colell, ${ }^{a}$ Rafael Müller, ${ }^{a}$ Bernhard Blümich ${ }^{a}$ and Stephan Appelt ${ }^{a b}$ \\ Received 31st December 2010, Accepted 4th February 2011 \\ DOI: 10.1039/c0an01048k
}

\begin{abstract}
Advances with para-hydrogen induced polarization open up new fields of applications for portable low-field NMR. Here we report the possibility of tracing drugs down to the micromolar regime. We could selectively polarize nicotine quantities similar to those found in one cigarette. Also less than $1 \mathrm{mg}$ of harmine, a drug used for treatment of Parkinson's disease, and morphine extracted from an opium solution were detectable after polarization with parahydrogen in single-scan ${ }^{1} \mathrm{H}$-experiments. Moreover, we demonstrate the possibility to selectively enhance and detect the ${ }^{1} \mathrm{H}$-signal of drug molecules with PHIP in proton rich standard solutions that would otherwise mask the ${ }^{1} \mathrm{H}$ NMR signal of the drug.
\end{abstract}

\section{Introduction}

Low-field NMR devices with permanent magnets and with electromagnets are inexpensive, mobile, and can be used in different applications, such as quality control, well-logging, and material science. In comparison to high-field NMR, low-field devices suffer from lower sensitivity compared to high-field NMR as the thermodynamic equilibrium magnetization is proportional to the magnetic field strength. However, the signal can be enhanced by hyperpolarizing the longitudinal magnetization to values beyond those attained in thermodynamic equilibrium by techniques like dynamic nuclear polarization $^{1-3}$ (DNP), spin exchange optical pumping ${ }^{4,5}$ (SEOP), spin polarization-induced nuclear Overhauser effect ${ }^{6,7}$ (SPINOE), and para-hydrogen induced polarization 4 (PHIP). In the past the use of PHIP was limited to hydrogenation reactions ${ }^{8-12}$ until it was shown that similar enhancements can be achieved by non-reactive, templatemediated contacts between para-hydrogen and target molecules, a method denoted as Signal Amplification by Reversible-Exchange (SABRE). ${ }^{13}$ Here, para-hydrogen associates with a transition metal complex, which acts as a template, and the hydrogenated complex adds to a substrate allowing the polarization to be transferred to the substrate by scalar coupling. The polarization transfer is only successful if the final complex of three partners is stable for a limited time only, meaning, that all three complex-forming partners

${ }^{a}$ Institute of Technical and Macromolecular Chemistry, RWTH Aachen University, Worringer Weg 1, 52074 Aachen, Germany. E-mail: stgloeggler@gmx.de; Tel: +492418020469

${ }^{b}$ Central Institute for Electronics, Research Center Jülich, WilhelmJohnen-Straße, 52428 Juelich, Germany.E-mail: st.appelt@fz-juelich.de; Tel: +492461613884 dissociate after a short time. Recently, it was shown that trace amounts as low as $12 \mathrm{nmol}$ of pyridine can be still detected using SABRE. ${ }^{14}$ Here, we report an application of low-field NMR combined with SABRE to detect traces of drugs, especially alkaloids, and the possibility to selectively enhance the signal of drug molecules in solutions where solvent and guest signals would overlap when nonselectively polarized in thermodynamic equilibrium, so that the drug signal could not be detected.

\section{Experimental section}

For the harmine sample preparation $1.0 \mathrm{mg}$ of catalyst $\left[\operatorname{Ir}(\mathrm{COD})\left(\mathrm{PCy}_{3}\right)(\mathrm{py})\right]\left[\mathrm{PF}_{6}\right]\left(\mathrm{Pcy}_{3}\right.$ : tris-cycohexyl-phosphine, py: pyridine) was dissolved in $0.4 \mathrm{ml}$ deuterated methanol- $\mathrm{d}_{4}$ and $0.7 \mathrm{mg}$ harmine were added. The second sample consisted of $25 \mathrm{mg}$ of nicotine and $1.0 \mathrm{mg}$ catalyst dissolved in $0.38 \mathrm{ml}$ deuterated methanol- $\mathrm{d}_{4}$. Morphine was extracted from an opium solution. The solution consisted of $1 \%$ morphine, 35\% ethanol and about $64 \%$ water. Water and ethanol were first evaporated by heating the solution, and the morphine was extracted afterwards using $5 \mathrm{ml}$ of deuterated methanol- $\mathrm{d}_{4}$. From that solution $0.4 \mathrm{ml}$ were filled into an NMR tube, and $1.0 \mathrm{mg}$ catalyst was added. A second sample with morphine was prepared by diluting $0.2 \mathrm{mg}$ of the earlier morphine solution with another $0.2 \mathrm{mg}$ deuterated methanol- $\mathrm{d}_{4}$. The two final samples contained $1.0 \mathrm{mg}$ and $0.5 \mathrm{mg}$ morphine to which again 1.0 mg catalyst was added.

The NMR experiments were carried out with a home-built NMR spectrometer at $3.9 \mathrm{mT}\left(166 \mathrm{kHz}{ }^{1} \mathrm{H}\right.$-resonance). NMR tubes containing the drugs to be investigated were connected to a liquid nitrogen cooled para-hydrogen setup, in which $50 \%$ of hydrogen were converted into para-hydrogen. In preparation of the measurement, 5 bar of hydrogen gas was supplied to the sample. The sample was then shaken inside the magnetic field to ensure proper dissolution of the para-hydrogen gas in the methanol-drug solution, and subsequently placed in the center of the $B_{0}$ coil. A proton spectrum was acquired with a single scan using a $90^{\circ}$ pulse. Afterwards the samples were thermally polarized inside a $2 \mathrm{~T}$ Halbach magnet, transported to the coil in less than a second, a proton spectrum was acquired with a single scan using a $90^{\circ}$ pulse.

\section{Results and discussion}

Three alkaloids were investigated: harmine, nicotine and morphine. Harmine belongs to the harmala alkaloids. It is used for medication of patients with Parkinson's disease and encephalitis. High dosages 
can cause hallucinations. Nicotine is the main proponent of the nicotiana alkaloids and is found in every part of the tobacco plant. ${ }^{15}$ The hyperpolarization of nicotine using SABRE and its detection in high magnetic fields was already demonstrated. ${ }^{13}$ We show that nicotine can be detected in low magnetic fields as well, which can be important for future low-field NMR applications because nicotine is the most widely spread addictive drug. Morphine is an opium alkaloid and occurs in huge amounts in opium poppy seeds. The most important effects of morphine on the human body are relief of pain and respiratory depression.

Small amounts of the drugs can be dissolved in methanol and after addition of catalyst polarized using SABRE. Fig. 1 depicts a comparison between the thermally prepolarized harmine samples at $B_{\mathrm{p}}=2 \mathrm{~T}$ and the harmine samples polarized with para-hydrogen at $B_{0}=3.9 \mathrm{mT}$.

The insets in (b) and (d) in all figures show an enhanced view at the beginning of the FID. It is remarkable that it is possible to polarize and detect as little as $0.7 \mathrm{mg}(3.3 \mu \mathrm{mol})$ of the substance. The amplitude of the hyperpolarized ${ }^{1} \mathrm{H}$ time domain signal is about twice larger than the amplitude of the thermally polarized signal and well above the noise level, which indicates that even smaller concentrations would be detectable. It is noted, that the hyperpolarized harmine signal appears in anti-phase, building up to a maximum before it decays to zero (see inset Fig. 1(d)). Attempts have been made to explain such signals for three- and four-spin systems ${ }^{16,17}$ but harmine is far more complex, and it is not clear why the antiphase signal arises. The frequency difference $\Delta \nu=0.64 \mathrm{~Hz}$ between the two peaks in Fig. 1(c) corresponds to the chemical shift difference of 4 ppm, which is the difference between the aromatic ring protons and the two methyl groups in the harmine molecule. This frequency difference cannot be resolved for the thermally prepolarized harmine sample in Fig. 1(a) due to line-narrowing effects and the antiphase structure in the SABRE case. In the following we made an attempt to understand the antiphase signal: The measured $\Delta \nu=0.64 \mathrm{~Hz}$ can be attributed to a chemical shift difference if the ${ }^{1} \mathrm{H}$-homonuclear J-couplings between the protons in the harmine molecule are substantially smaller than $\Delta \nu$. This is the case for protons A-E (Fig. 1) coupled by ${ }^{5} J_{\mathrm{H}, \mathrm{H}} \approx 0.1$
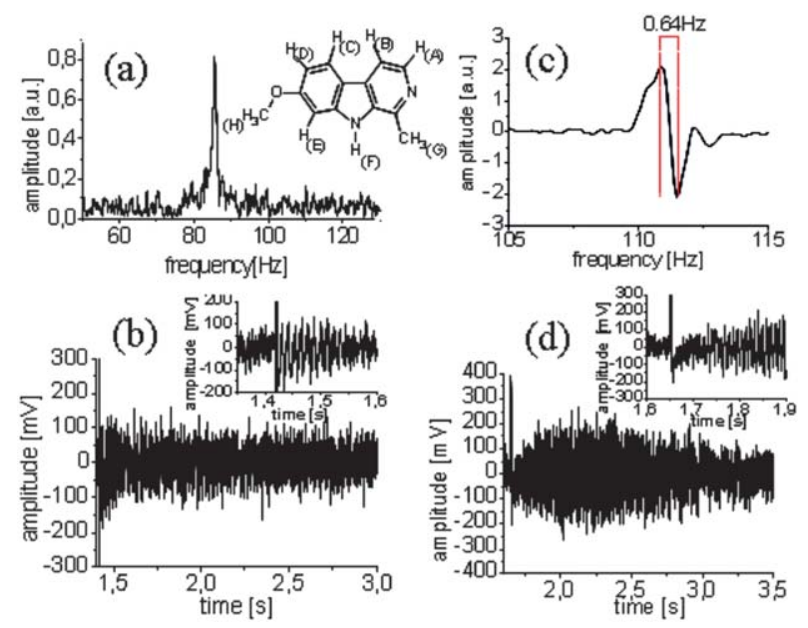

Fig. 1 Single scan ${ }^{1} \mathrm{H}$ harmine experiments. Spectrum (a) and free induction decay (FID, b) of the thermally polarized harmine sample at $B_{\mathrm{p}}=2 \mathrm{~T}$. Spectrum (c) and FID (d) of harmine after polarization with parahydrogen. The FID and the spectrum clearly show an antiphase signal.
$\mathrm{Hz}<\Delta \nu$ to the two methyl groups $\mathrm{G}$ and $\mathrm{H}$ but not for the pairs $\mathrm{A}-\mathrm{B}$ or $\mathrm{C}-\mathrm{D}$, for which ${ }^{3} J_{\mathrm{H}, \mathrm{H}} \approx 7 \mathrm{~Hz}>\Delta \nu$ is valid. A more detailed quantitative analysis is necessary for the inverse strong coupling regime $\left(\Delta \nu<J_{\mathrm{H}, \mathrm{H}}\right)$ in low magnetic fields.

Also very small amounts of morphine can be detected (Fig. 2). For a $1 \mathrm{mg}$ sample, the signal amplitude of the hyperpolarized sample is as large as thermal equilibrium magnetization of $2 \mathrm{~T}$, whereas the signal-to-noise ratio of the hyperpolarized morphine signal is larger than for the thermal polarized sample. Even after further dilution by a factor of two, the signal from $1.7 \mu \mathrm{mol}$ can still be recorded with the SABRE method. Regarding the nicotine sample (Fig. 3), the signal amplitudes in the time domain of the thermally polarized and the hyperpolarized sample differ by the same factor as for the morphine. When comparing the hyperpolarized with the thermally polarized nicotine signal it is noted, that in the latter case two peaks are

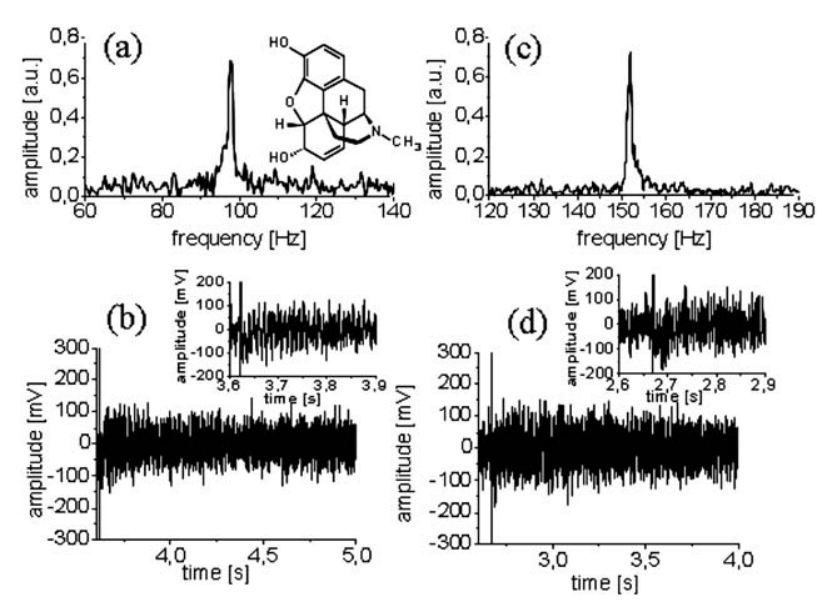

Fig. $2{ }^{1} \mathrm{H}$ morphine experiments measured with a single scan. Spectrum (a) and free induction decay (b) of the thermally polarized morphine sample at $B_{\mathrm{p}}=2 \mathrm{~T}$. Spectrum (c) and free induction decay (d) of morphine after polarization with para-hydrogen.
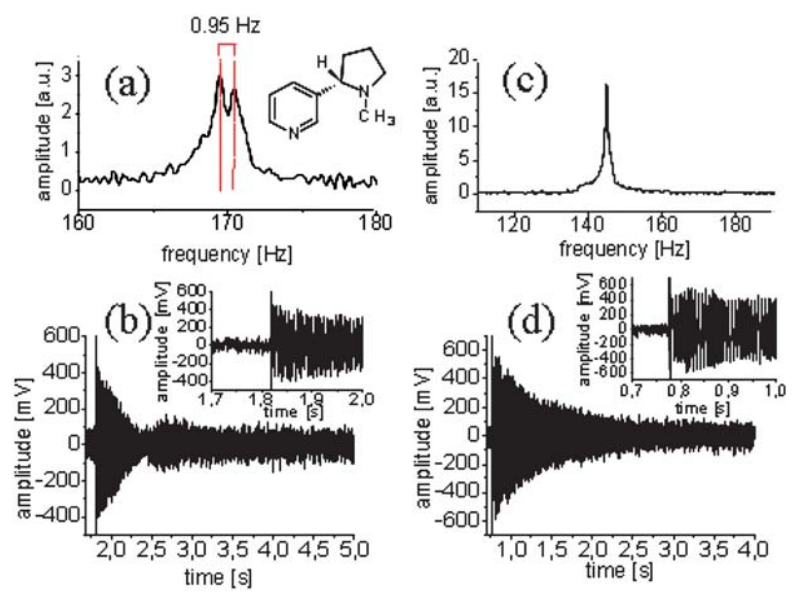

Fig. $3{ }^{1} \mathrm{H}$ nicotine experiments measured with a single scan. Spectrum (a) and free induction decay (b) of the thermally polarized nicotine sample. Two peaks that belong to the aromatic and aliphatic part of nicotine with a chemical shift difference of $0.95 \mathrm{~Hz}(5.7 \mathrm{ppm})$ are observed. Spectrum (c) and free induction decay (d) of morphine after polarization with para-hydrogen. Only one peak is observed because only the nitrogen containing aromatic part becomes polarized. 

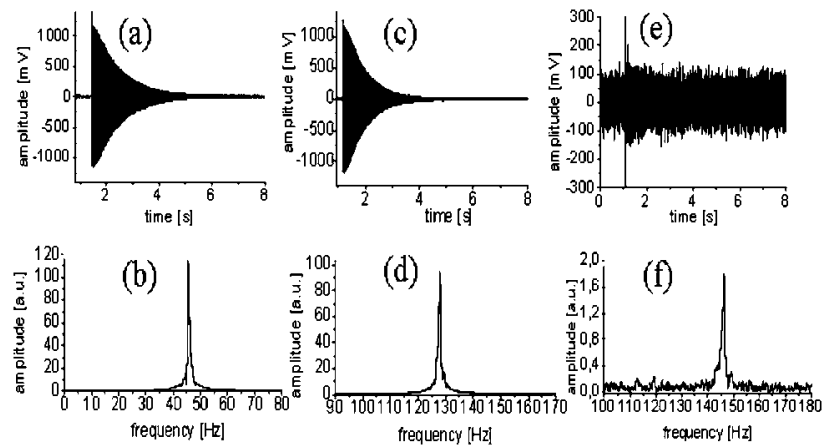

Fig. 4 Selective enhancement of nicotine in methanol. ${ }^{1} \mathrm{H}$ FID and spectrum of $0.4 \mathrm{ml}$ methanol after prepolarizing at $B_{\mathrm{p}}=2 \mathrm{~T}$ (a and b), methanol $(0.4 \mathrm{ml})$ and nicotine $(4 \mathrm{mg})\left(\mathrm{c}\right.$ and d) prepolarized at $B_{\mathrm{p}}=2 \mathrm{~T}$, and ${ }^{1} \mathrm{H}$ FID and spectrum of $4 \mathrm{mg}$ nicotine in $0.4 \mathrm{ml}$ methanol selectively enhanced with SABRE (e and f).

observed, which are separated by $0.95 \mathrm{~Hz}$ or $5.7 \mathrm{ppm}$. These two peaks arise from the chemical shift differences of the aromatic and aliphatic groups in nicotine. Our understanding of the polarization transfer with SABRE is that polarization is transferred only via nitrogen atoms, which possess a free orbital reaching out into space close to the protons without posing a steric barrier to the temporary addition of the catalyst. In nicotine only the nitrogen in the aromatic ring fulfils this condition (see Fig. 3(a)) and therefore only the protons in the ring are polarized and detectable resulting in just one observable peak. Harmine and morphine also contain aromatic and aliphatic parts but we were not able to resolve them at our magnetic field strength of $3.9 \mathrm{mT}\left(166 \mathrm{kHz}{ }^{1} \mathrm{H}\right.$ frequency).

In addition to achieving hyperpolarization as such, the selectivity of the SABRE method is another outstanding advantage, as small amounts of target molecules with suitable nitrogen atoms can be selectively polarized and detected in complex mixtures. This is demonstrated in Fig. 4 with a methanol-nicotine mixture. Fig. 4a and $\mathrm{b}$ show the thermally polarized ${ }^{1} \mathrm{H}$ signal of $0.4 \mathrm{ml}$ pure methanol. If 4 $\mathrm{mg}$ of nicotine are added and the spectrum is recorded after thermal polarization, it is not possible to observe the nicotine component (Fig.4c and d) because the proton signal of methanol is too large, while with SABRE, the nicotine signal is selectively enhanced (Fig.4e and f). It should be noted that the frequency differences in Fig. 4b, $\mathrm{d}$ and $\mathrm{f}$ arise from the fact that the used spectrometer is an electromagnet and therefore $B_{0}$ is not perfectly stable and experiences a small drift within time, resulting in increasing off-resonance frequencies of the NMR signal.

\section{Conclusion}

We have demonstrated the application of low-field (mT) NMR and SABRE to the detection of small amounts of drugs. For harmine and morphine it was possible to detect parts of milligrams (micromol regime) of a substance. A few milligrams of nicotine, an amount that corresponds to one found in a single cigarette, could be detected in a single scan with low-field NMR setup. One outstanding feature of this method is its selectivity in enhancing the signals of the target molecules selected by the catalyst in complex mixtures as demonstrated for a methanol-nicotine solution. We like to state that the sensitivity limit of the presented method for tracing drugs has not yet been reached. For example, all the experiments were carried out with a hydrogen gas mixture containing just 50\% para-hydrogen. By further cooling it is possible to reach $100 \%$ conversion of orthohydrogen into para-hydrogen resulting in a further increase of polarization. In combination with a mobile NMR spectrometer, which has a high field homogeneity and an optimized sensitivity, it is conceivable that mobile NMR machines can serve in the future to identify even smaller amounts of drugs traces at high accuracy and differentiate between them.

\section{References}

1 A. Abragam and M. Goldman, Rep. Prog. Phys., 1978, 41, 395-467.

2 M. E. Halse and P. T. Callaghan, J. Magn. Reson., 2008, 195, 162168.

3 M. D. Lingwood, I. A. Ivanov, A. R Cote and S. Han, J. Magn. Reson., 2010, 204, 56-63.

4 W. Happer, Rev. Mod. Phys., 1972, 44, 169-249.

5 S. Appelt, A. B. Baranga, C. J. Erickson, M. V. Romalis, A. R. Young and W. Happer, Phys. Rev. A, 1998, 58, 1412-1439.

6 G. Navon, Y. Q. Song, T. Room, S. Appelt, R. E. Taylor and A. Pines, Science, 1996, 271, 1848-1851.

7 S. Appelt, F. W. Häsing, S. Baer-Lang, N. J. Shah and B. Blümich, Chem. Phys. Lett., 2001, 348, 263-269.

8 J. Natterer and J. Bargon, Prog. Nucl. Magn. Reson. Spectrosc., 1997, 31, 293-315.

9 P. Hübler, R. Giernoth, G. Kümmerle and J. Bargon, J. Am. Chem. Soc., 1999, 121, 5311-5318.

10 S. Aime, D. Canet, W. Dastru, R. Gobetto, F. Reineri and A. J. Viale, Phys. Chem. A, 2001, 105, 6305-6310.

11 L. S. Bouchard, S. R. Burt, M. S. Anwar, K. V. Kovtunov, I. V. Koptyug and A. Pines, Science, 2008, 319, 442-445.

12 K. V. Kovtunov, I. E. Beck, V. I. Bukhtiyarov and I. V. Koptyug, Angew. Chem., 2008, 120, 1514-1517.

13 R. W. Adams, J. A. Aguilar, K. D. Atkinson, M. J. Cowley, I. P. P. Elliott, S. B. Duckett, G. G. R. Green, I. G. Khazal, J. Lopez-Serrano and D. C. Williamson, Science, 2009, 323, 17081711.

14 Q. Gong, A. Gordji-Nejad, B. Blümich and S. Appelt, Anal. Chem., $2010,82,7078-7082$.

15 A. Fallert-Müller, Lexikon der Biochemie Band 1 und 2, Spektrum Verlag, Heidelberg, 1999/2000.

16 K. L. Ivanov, A. V. Yurkoskaya and H.-M. Vieth, J. Chem. Phys., 2008, 128, 154701.

17 R. W. Adams, S. B. Duckett, R. A. Green, D. C. Williamson and G. G. R. Green, J. Chem. Phys., 2009, 131, 194505. 